\title{
A new generation of commercial brain surgery robot clinical application research
}

Feng Yin ( $\square$ yinf897@163.com )

Aerospace Center Hospital

\section{Hui Chen}

Aerospace Center Hospital

Xin Yan

Aerospace Center Hospital

Sheng-chao Ding

Aerospace Center Hospital

Ming-Ming Zhao

Aerospace Center Hospital

Shou-Ming Cao

Aerospace Center Hospital

Tong Li

Aerospace Center Hospital

Chun-Juan Yang

Aerospace Center Hospital

Ying Liu

Aerospace Center Hospital

Yu Huang

Beijing University of Aeronautics and Astronautics

Feng Zhao

Beijing University of Aeronautics and Astronautics

\section{Ya-Ming Wang}

The Xuanwu Hospital

\section{Research Article}

Keywords:

Posted Date: March 3rd, 2022

DOI: https://doi.org/10.21203/rs.3.rs-1355563/v1 
License: (c) (i) This work is licensed under a Creative Commons Attribution 4.0 International License. Read Full License 


\title{
A new generation of commercial brain surgery robot clinical application research
}

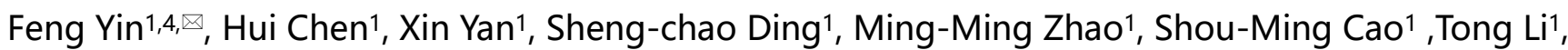
Chun-Juan Yang ${ }^{1}$, Ying Liu1 ${ }^{1}$, Yu Huang ${ }^{2,4}, \bowtie$, Feng Zhao², Ya-Ming Wang $3,4, \bowtie$

1. Department of Neurosurgery, Aerospace Center Hospital, Beijing, China. ${ }^{2}$. Department of Biomechanics and Mechanobiology, Ministry of Education Beijing Advanced Innovation Center for Biomedical Engineering School of Biological Science and Medical Engineering, Beijing University of Aeronautics and Astronautics, Beijing, China.

3. Department of Neurosurgery, The Xuanwu Hospital, Beijing, China. ${ }^{4}$ These author contributed equally: Feng Yin, Yu Huang and Ya-Ming Wang. 『email: yinf897@163.com; youth191954@qq.com; yamingwang@sohu.com;

\section{【 Abstract 】}

To evaluate a new generation of commercial robotic (CR) the clinical practicability of stereotactic rob otic system, operating accuracy and operation safety. We analyzed retrospectively in 08, 2018-2020 - Jun e, during the application of CR type brain surgery robot system implementation frameless stereotactic bra in surgery clinical data of 120 patients, observe clinical practicability, positioning accuracy and safety. Clin -ical successful completion of the frameless positioning brain surgery in 120 cases, the procedure goes well, no serious complications. Including registration and operation stage, the average operation time was 23.5 to $80.2 \mathrm{~min}$. To the postoperative target accuracy evaluation, the results show that target error in 1 . 36 and $0.42 \mathrm{~mm}, 4$ cases of patients with brain damaged kernel group, using three nail operation head $\mathrm{f}$ rame fixed Mayfield, use solid coupling device to robot and heads fixed after surgery, results show that $t$ he target error in 0.92 and $0.21 \mathrm{~mm}$, meet the operation requirements. The new generation of CR stereo tactic robotic system has great improvement compared with the previous generation, which can apply to all types of stereotactic surgery at present, to enhance the practical operation, the structure design more reasonable, integrated practical operation, positioning precision and convenient operation, meet the deman $\mathrm{d}$ of clinical application.

$\begin{array}{ll}\text { Abbreviations } & \\ \text { CR } & \text { commercial robotic } \\ \text { HOZ MEDICAL } & \text { Horizon Microport Medical Technology (Beijing) CO., LTD } \\ \text { HICH } & \text { hypertensive cerebral hemorrhage } \\ \text { CNS } & \text { central nervous system } \\ \text { LC } & \text { Langerhans cell } \\ \text { DBS } & \text { deep brain electrode stimulation } \\ \text { SEEG } & \text { stereoelectroencephalography } \\ \text { VAS SCORE } & \text { The visual pain score }\end{array}$

Modern stereotactic neurosurgery is developing in the direction of refinement and programming. Frameless stereotactic surgery represents this development trend and has a very broad application field[1-3]. Since the PUMA robot was first used in neurosurgery in 1985 [4], medical robotics technology has achieved significant development, from the early industrial robot platform to the current dedicated robot, from the early large and complex structure to the current small modular The structure, from the early simple functions to the current multifunctional, remote surgical operations, medical surgical robotics has shown its own development characteristics, and has formed a cutting-edge academic field[5-11]

At this stage, robots are mainly used to assist positioning in the field of neurosurgery. They can be integrated into the surgical workflow as an active or passive system. The doctor is still the main body in the operation. In the active system, the robot can actively complete the walking position of each articulated arm according to the plan of the surgeon and reach the surgical posture position ${ }^{[9]}$. Currently in China, such products are represented by ROSA, Remebot and Huake Precision. In the passive system, the computer calculates the 
value of each articulated arm, and the surgeon manually adjusts the position of each articulated arm to reach the surgical posture position. At present, this type of robot is represented by the CAS-R-2 robot made by Horizon Microport Medical Technology (Beijing) CO., LTD ( Hoz Medical) in China.

Although the robotic surgery system has brought great convenience to the clinic, it still encounters $s$ ome problems in practical applications. For example, the robotic equipment occupies a large space and has poor flexibility and mobility; the head frame is still used during the operation, which will cause proble ms for the patient. Trauma; long registration time for surgery; possibility of relative displacement; mutual i nterference of equipment in the surgical area, especially during posterior fossa surgery; long learning cur-ve, complicated operation process, etc.

The CAS-R-2 stereotactic robot system obtained the product registration certificate in 2000 and was the first robot system in China to start clinical application. Compared with the active arm robot, the machine is small and flexible, has strong maneuverability, simple operation, and short learning curve. So far, the robot system has completed clinical applications in 10568 patients [12-17]. However, with the advancement of technology, some shortcomings of the robot system have also been reflected, which limits its further clinical application.

Insufficiency of mechanical design: (1)The counterweight of the machine is lighter, and the load of the mechanical arm is weak, which will affect the service life; (2)The mechanical arm has weak tolerance to drilling; (3)The control box is not modularized, and the equipment is stable during transportation. Sex has an impact. Disadvantages in clinical use: (1)Fixing methods are cumbersome and poor in stability. The lack of a fixed connection device between the equipment and the operating table is prone to relative displacement, which affects the accuracy of the operation, thus limiting the scope of surgical application. At present, the system is mainly used for cerebral hemorrhage and brain biopsy and other visual target operations. (2)The aging of the planning software cannot meet the needs of three-dimensional visualization in path planning, which affects the accuracy of the operation; (3)The single planning module and no functional operation module are set, which affects the versatility of the machine. Surgical robots will prolong with the use of time. If they are not maintained and maintained in a timely manner, the positioning accuracy will be affected CR type passive arm brain surgery robot is easier to maintain than traditional active arm, easy to use, and has joint locking, which can effectively ensure the navigation accuracy during surgery.

This study aims to improve the software and hardware of the CAS-R-2 robot according to the needs of the clinical development of neurosurgery and developed a new generation of $\mathrm{CR}$ robot equipment to make it practical and practical. Accuracy and safety are more in line with clinical needs.

\section{MATERIALS AND METHODS}

Patients. The cases came from 120 patients who underwent robot-assisted stereotactic surgery admitted to the Aerospace Center Hospital from 2018.08 to 2020.06. Among them, 63 were males and 57 were females; they were 3 to 89 years old, with an average of 48 years old. The types of operations included: intracerebral lesion biopsy in 82 cases; intracerebral hematoma emptying in 20 cases; OMMAYA capsule implantation in 7 cases; brain abscess aspiration in 5 cases; brain mass destruction in 4 cases, and stereotactic-guided resection of small lesions in the brain in 2 cases. This study was approved by the Ethic Committee for Drug Clinical Trials, Aerospace Center Hospital (EC2018-027). All methods were carried out in accordance with relevant guidelines and regulations. Informed consent was obtained from all subjects (participants of age greater than 16 years) and/or their legal guardian(s).

Data measurements. The new stereotactic brain surgery system (CR type) consists of a robotic arm, a computer, a special instrument cart, surgical navigation software, end tools and probes (Figure 1). The system integrates surgical planning, positioning and navigation modules, which simplifies surgical procedures while ensuring surgical accuracy. The new generation CR brain surgery robot system optimizes the mechanical design: (1)The counterweight of the machine is increased, and the load of the mechanical arm is increased, which can extend its service life; (2)The resistance of the mechanical arm to drilling is improved; (3)The control box Carry out modular control to reduce the impact of transportation on equipment stability. Through the establishment of a three-dimensional visualization model, real-time and interactive browsing can be carried out, which is convenient 
for the surgeon to complete the selection and planning of the surgical path, and greatly reduces the preoperative preparation and planning time.

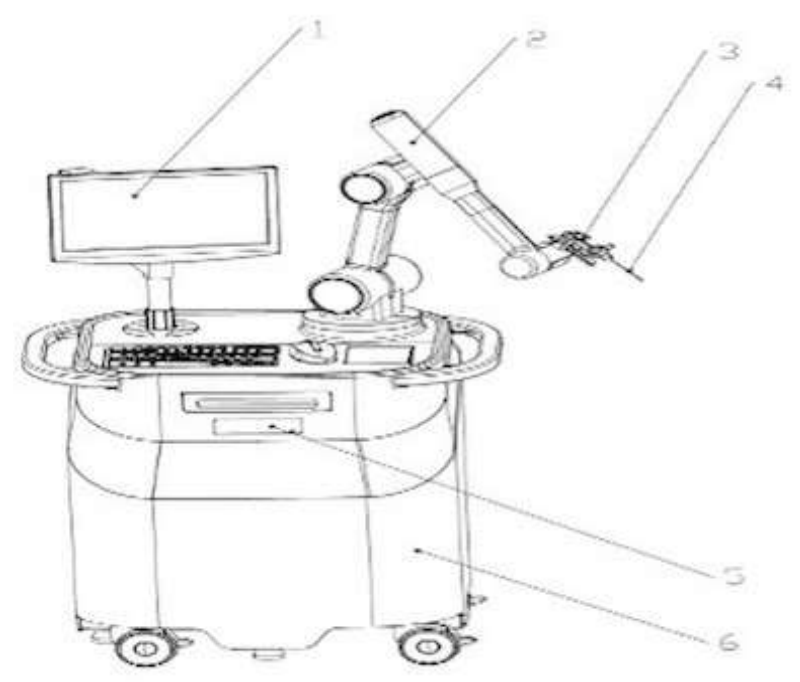

Figure 1. New type CR brain surgery robot system. (1. Surgical navigation software 2. Robot arm 3. End tool 4. Probe 5. Computer 6. Special instrument vehicle)

The analysis of potential failure modes of the new CR brain surgery robot is carried out, and the integration of manipulator, surgical navigation software and equipment is analyzed for risk analysis from multiple factors of biology, physics, and chemistry. Main hazards: (1)The equipment slips. (2)The distance between the equipment keys is too small to operate or mis operation. (3)The environmental condition of the equipment exceeds the limit. (4) Wrong connection or operation of equipment.

Possible damages caused by the above hazards: (1)Damage to equipment or injury to surrounding personnel. (2)The equipment cannot be used normally or the equipment is damaged. (3)The product is damaged and cannot be used normally. (4) Equipment damage or personal injury. Measures taken against its hazards: (1) The equipment uses casters with a locking structure, and the manual clearly specifies that the casters are locked during use. (2)Ensure operation space between the keys on the control panel. (3)Through design improvement, the product meets EMC requirements. (4) Add function labels to the equipment to make it clear that each label conforms to the meaning. The new CR brain surgery robot has completed the verification of measures taken against the hazards through functional tests and experimental tests, and the final overall risk is acceptable without affecting its safety and effectiveness.

\section{Surgical methods}

(1) Cranial MRI scan 1-2 days before surgery. (2)Locating marks: On the day of surgery, attach 4 to 6 location marks to the patient's head (around the lesion), and perform a head CT scan (bone MARK for nuclear damage, and scalp MARK for the rest). Patients with acute intracerebral hemorrhage only undergo head CT scan. (3)Robot preparation: including the deployment and spatial positioning of the robot. (4)Surgery planning: Input head CT/MRI scan results into the computer through computer network or mobile storage device, perform image fusion, the surgeon (local or remote) outlines the lesion, determines the surgical target and puncture path, and computerassisted calculation of the target Point coordinates, showing the puncture path and 3D imaging. (5)Position and anesthesia: Choose different positions according to the diseased location, such as supine position and lateral position. Reasonable choice of anesthesia, most patients use intravenous anesthesia or intubation anesthesia, for intraoperative tests such as Parkinson's and intractable pain patients can complete the operation under local anesthesia. (6)Fixed: Use a shaping pillow (Figure 2) or a surgical head frame combined with a fixed connection device to ensure the relative position of the patient's head, body position and the intelligent robotic arm are fixed. (7) Surgery simulation: According to the target coordinates and puncture path set by the computer, perform the operation simulation; prepare for the operation after the simulation is successful. (8) Skull drilling: first place the protective drill sleeve in the guide clamp of the carriage and the ruler slider, and then insert the fine drill through 
this drill sleeve. Reach the scalp and inject local anesthetics there. After setting the protection depth of the drill skull, use a DC electric drill or a hand drill to drive a fine drill to drill through the skull at the point of entry. At this time, only the skull needs to be drilled. Be careful not to get into the brain. (9) Puncture needle: Take out the fine drill and drill sleeve, replace the corresponding protective sleeve, and then insert the puncture trocar. The surgeon holds the upper end of the trocar with his hand and uses the tip of the oblique incision at the lower end of the needle sleeve to poke the meninges in the skull at the point where it enters the skull. After that, various corresponding surgical operations were performed according to the condition of the disease.
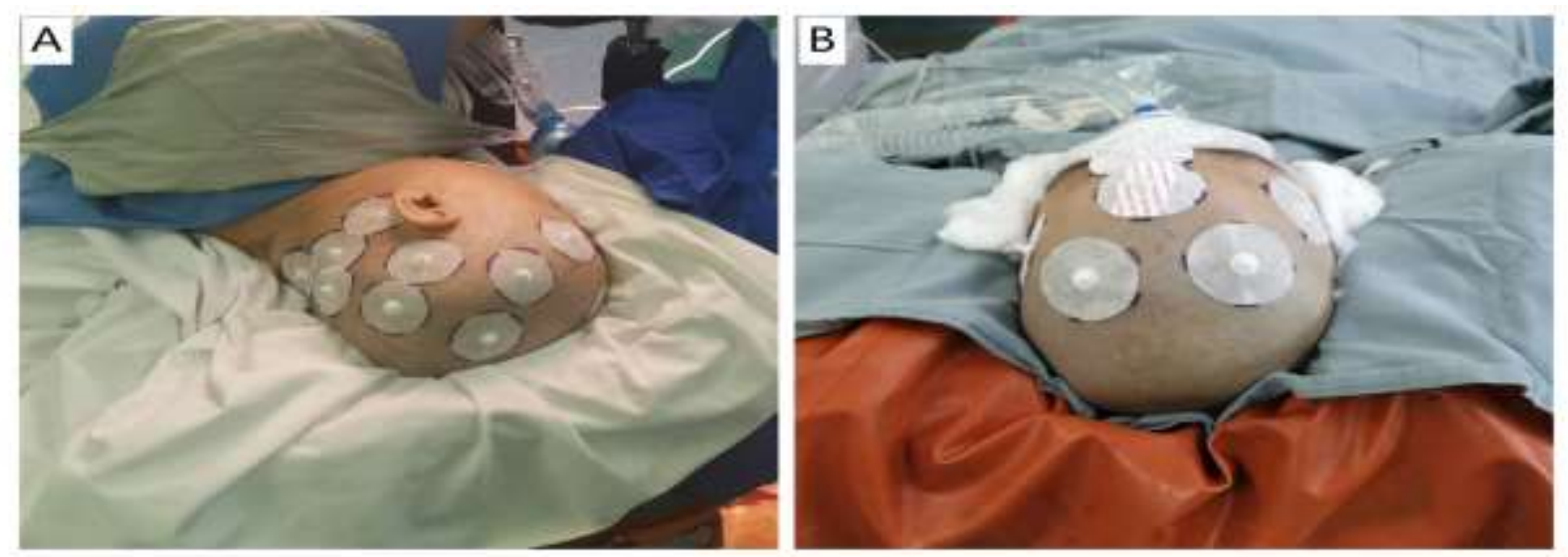

Figure 2. Shaping pillow fixation. A. Fixed lateral position; B. Orthostatic fixation

Statistical analysis. In order to evaluate the accuracy of the target during the operation of plastic pillow fixation, we verified the accuracy of target positioning for all biopsy patients. The method is as follows: in the postoperative $\mathrm{CT} / \mathrm{MRI}$ scan image, measure and calculate the Euclidean distance between the planned target point and the tissue point of the biopsy needle tip before the operation. The Euclidean distance between two points is defined as the square sum and the square root of the coordinate difference of each point on the three coordinate axes of $x, y$ and $z$. When a patient is scanned by imaging equipment such as CT/MRI, the formed DICOM image data includes the definition of the coordinate system. In the robotic surgical navigation system, we call the coordinate system composed of DICOM data as the model coordinate system. Since the DICOM data of the CT/MRI threedimensional scan imaging before and after the patient has its own independent model coordinate system, the Euclidean distance of the target cannot be directly calculated in the postoperative CT/MRI scan image, and the coordinate system conversion is required. Convert the target point coordinates in the postoperative model coordinate system to the coordinates in the preoperative model coordinate system.

We identified 3 distinct anatomical landmarks in the preoperative and postoperative CT/MRI images (such as ethmoid comb, anterior clinoid process of sphenoid bone (left), anterior clinoid process of sphenoid bone (right), (Figure 3). Suppose the three anatomical structure points are points C1, C2, C3.

The anatomical coordinate system is established: $\mathrm{C} 1$ is the origin, $\mathrm{C} 1, \mathrm{C} 2$, the connection line is the $\mathrm{x}$ axis, the plane perpendicular to $\mathrm{C} 1, \mathrm{C} 2, \mathrm{C} 3$ is the $\mathrm{z}$ axis, and the $\mathrm{x}$ and $\mathrm{z}$ axes are perpendicular to the $\mathrm{y}$ axis. That is $\vec{x}=\operatorname{norm}\left(\overrightarrow{C_{2} C_{1}}\right), \vec{z}=\operatorname{norm}\left(\vec{x} \times \overrightarrow{C_{3} C_{1}}\right), \vec{y}=\vec{z} \times \vec{x}$. Where $\overrightarrow{C_{2} C_{1}}$ is the vector from point $\mathrm{C} 2$ to point $\mathrm{C} 1, \overrightarrow{C_{3} C_{1}} \mathrm{~S}$ the vector from point $\mathrm{C} 3$ to point $\mathrm{C} 1$, norm normalizes the vector to a unit vector. Then the matrix transformed from the model coordinate system of the CT image to the anatomical coordinate system is $\mathrm{M}=\left[\begin{array}{cccc}\vec{x} & \overrightarrow{\mathrm{y}} & \overrightarrow{\mathrm{z}} & C_{1} \\ 0 & 0 & 0 & 1\end{array}\right]^{-1}$, which is the point under the original image coordinate system $\overrightarrow{P_{I}}$, The formula for converting to anatomical coordinate system point $\overrightarrow{P_{A}}$ is: $\overrightarrow{P_{A}}=M \times \overrightarrow{P_{I}}$. 

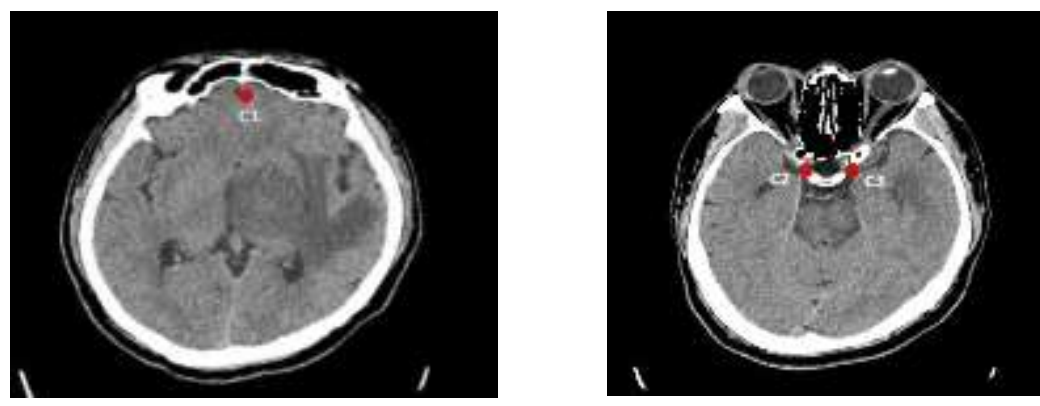

Figure 3. Preoperative and postoperative CT/MRI images to identify three distinct anatomical landmarks (such as ethmoid comb, left sphenoid anterior clinoid process, right sphenoid anterior clinoid process).

Obtain the planned target coordinate $\mathrm{T} 1$ in the preoperative image (CT/MRI) and solve its coordinate in the anatomical coordinate system as $\vec{P}_{1}=M * \vec{T}_{1}$; ; obtain the planned target coordinate in the preoperative image (CT/MRI) T2, solve its coordinate in the anatomical coordinate system as $\vec{P}_{2}=M * \vec{T}_{2}$ 。. In the same model coordinate system, the calculation of the distance between the planned target point and the actual target point is the navigation positioning error: $\Delta=\sqrt{\left(x_{1}-x_{2}\right)^{2}+\left(y_{1}-y_{2}\right)^{2}+\left(z_{1}-z_{2}\right)^{2}}$

\section{RESULTS}

In this study, 120 cases of neurosurgical robot-assisted stereotactic brain surgery were successfully $p$ erformed. All were successfully positioned at one time. The overall surgical success rate was $100 \%$, the early clinical transient complication was $2.5 \%$, and there were no serious surgical complications. The aver age operative time was $23.5-80.2 \mathrm{~min}$, including the registration stage and surgical stage (Table 1).

\begin{tabular}{|c|c|c|c|c|c|}
\hline Surgical approach & Number of cases & $\begin{array}{l}\text { Average operating ti } \\
\text { me (minutes) }\end{array}$ & $\begin{array}{l}\text { Accuracy } \\
(\mathrm{mm})\end{array}$ & $\begin{array}{l}\text { Symptomatic /Asymptomatic } \\
\text { hemorrhage } \\
\text { (n) }\end{array}$ & $\begin{array}{l}\text { Average follow-up } \\
\text { time (month) }\end{array}$ \\
\hline Biopsy & 82 & 23.5 & $1.36 \pm 0.42$ & $3 / 2$ & 3.2 \\
\hline $\begin{array}{l}\text { Catheterization and } d \\
\text { rainage of } \mathrm{HICH}\end{array}$ & 20 & 21.6 & & None & 6.8 \\
\hline $\begin{array}{l}\text { Catheter drainage for } \\
\text { brain abscess }\end{array}$ & 7 & 24.8 & & None & 7.2 \\
\hline $\begin{array}{l}\text { Ommaya capsule imp } \\
\text { lantation }\end{array}$ & 5 & 32.3 & & None & 9.2 \\
\hline $\begin{array}{l}\text { Damage to the inner } \\
\text { core of the brain }\end{array}$ & 4 & 59.3 & $0.92 \pm 0.21$ & None & 6.3 \\
\hline $\begin{array}{l}\text { Stereotactic guided re } \\
\text { section of small lesio } \\
\text { ns in the brain }\end{array}$ & 2 & 80.2 & & None & 12.8 \\
\hline
\end{tabular}

Table 1. Summary of results of 120 cases of robot-assisted surgery.

(HICH: hypertensive cerebral hemorrhage)

Biopsy of 82 deep brain lesions included 58 cases of cerebral hemisphere lesions, 17 cases of brain stem lesions, 4 cases of sellar lesions and 3 cases of pineal lesions (Figure 4). All cases received clear pathological diagnosis, pathological confirmed for glioma (52 cases), lymphoma (15 cases), demyelinating disease (5 cases), cerebral metastatic carcinoma (5 cases), inflammatory granuloma (2 cases), seminoma ( 1 case), Langerhans cell hyperplasia ( 1 case) and primary central nervous system vasculitis (1 case) (Table 2). 
Table 2. Types of diagnosis of 82 biopsy patients.

\begin{tabular}{lr}
\hline Diagnosis & $\begin{array}{c}\text { Number of } \\
\text { people }\end{array}$ \\
\hline Glioma & 52 \\
Lymphoma & 15 \\
Demyelinating disease & 5 \\
Brain metastases & 5 \\
Inflammatory granuloma & 2 \\
Seminoma & 1 \\
LC hyperplasia & 1 \\
Primary vasculitis of CNS & 1 \\
Sum & 82 \\
\hline
\end{tabular}

(CNS: central nervous system; LC: Langerhans cell)

One of the biopsy patients had moderate bleeding at the biopsy site, which improved after treatment. A small amount of bleeding occurred in the operation area in 2 cases with no obvious symptoms. Exce pt for one case of moderate bleeding, the rest of the patients could be discharged from the hospital $3 \mathrm{~d}$ ays after surgery or undergo the next step of treatment. 5 cases of deep brain abscess were punctured and placed. $12-35 \mathrm{ml}$ of pus was aspirated, and antibiotics were injected to flush the abscess cavity 1 to 2 times a day. The drainage tube was pulled out 3 days later, and systemic antibiotic treatment was con tinued for 4 to 8 weeks. The 5 cases of patients were cured and discharged. In 20 cases of brainstem and basal ganglia hematomas, $4 \mathrm{ml} 23 \mathrm{ml}$ of blood was drawn out (accounting for $60 \% \sim 80 \%$ of the total hematoma) during operation, and drainage was placed for 3 5 days after surgery, and 30,000 50,000 un its of urokinase were injected into the hematoma cavity. 4 cases of patients with brainstem hemorrhage and 2 cases of patients with supratentorial hematoma died eventually due to severe symptoms on admis sion. The remaining patients with cerebral hemorrhage had satisfactory emptying of the hematoma. 4 cas es of cystic craniopharyngioma and 1 case of cystic recurrence of glioma were treated with OMMAYA ca psule implantation. The operation was smooth, the fluid suction of the capsule was satisfactory, and the next treatment was successfully carried out. 3 cases of patients with Parkinson's disease and 1 patient $\mathrm{w}$ ith thalamic pain were given damage to the inner core of the brain (Figure 5), and the surgical target $w$ as accurate. Following postoperative follow-up, the UPDRS off-period scores of 3 patients with Parkinson' s disease improved significantly (see Table 3).

Table 3. UPDRS score.

\begin{tabular}{ccccc}
\hline & Baseline & 1Months & 3 Months & 6 Months \\
\hline 1 & 56 & 35 & 37 & 37 \\
2 & 54 & 24 & 22 & 26 \\
3 & 41 & 8 & 8 & 10 \\
\hline
\end{tabular}

The visual pain score (VAS score) of patients with thalamic pain decreased from 8-9 points before $s$ urgery to 2-3 points after surgery, and the quality of life was significantly improved. Plastic pillow fixation was adopted for the surgery of relatively large visual lesions (such as biopsy, cerebral hemorrhage and c erebral abscess). In order to evaluate the reliability of this fixation method, we performed postoperative ta rget accuracy on 82 biopsy patients. Evaluation results showed that the target error was $1.36 \pm 0.42 \mathrm{~mm}$. In 4 cases of patients with brain core mass destruction, the patients' heads were fixed with Mayfield thr ee-nail surgical head frame during the operation. After the robot was fixed to the surgical head frame wit $\mathrm{h}$ a fixed-link device, the operation was performed under local anesthesia. The postoperative target accur acy evaluation results showed that the target error was $0.92 \pm 0.21 \mathrm{~mm}$. 

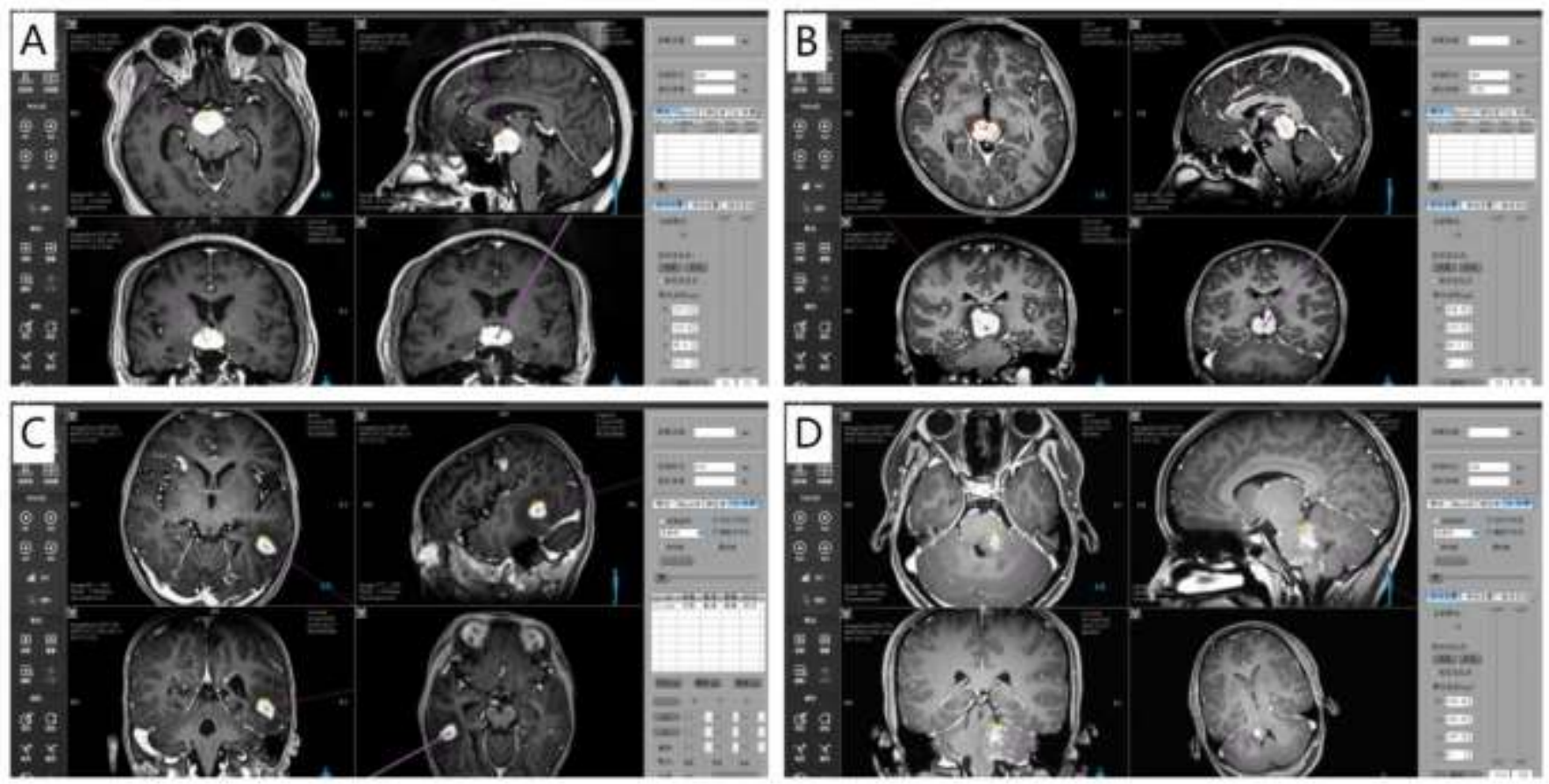

Figure 4. Robotic biopsy operation for different lesions in the brain. A Saddle lesions; B Pineal lesions; C Supratentorial lesions; D Brain stem lesions
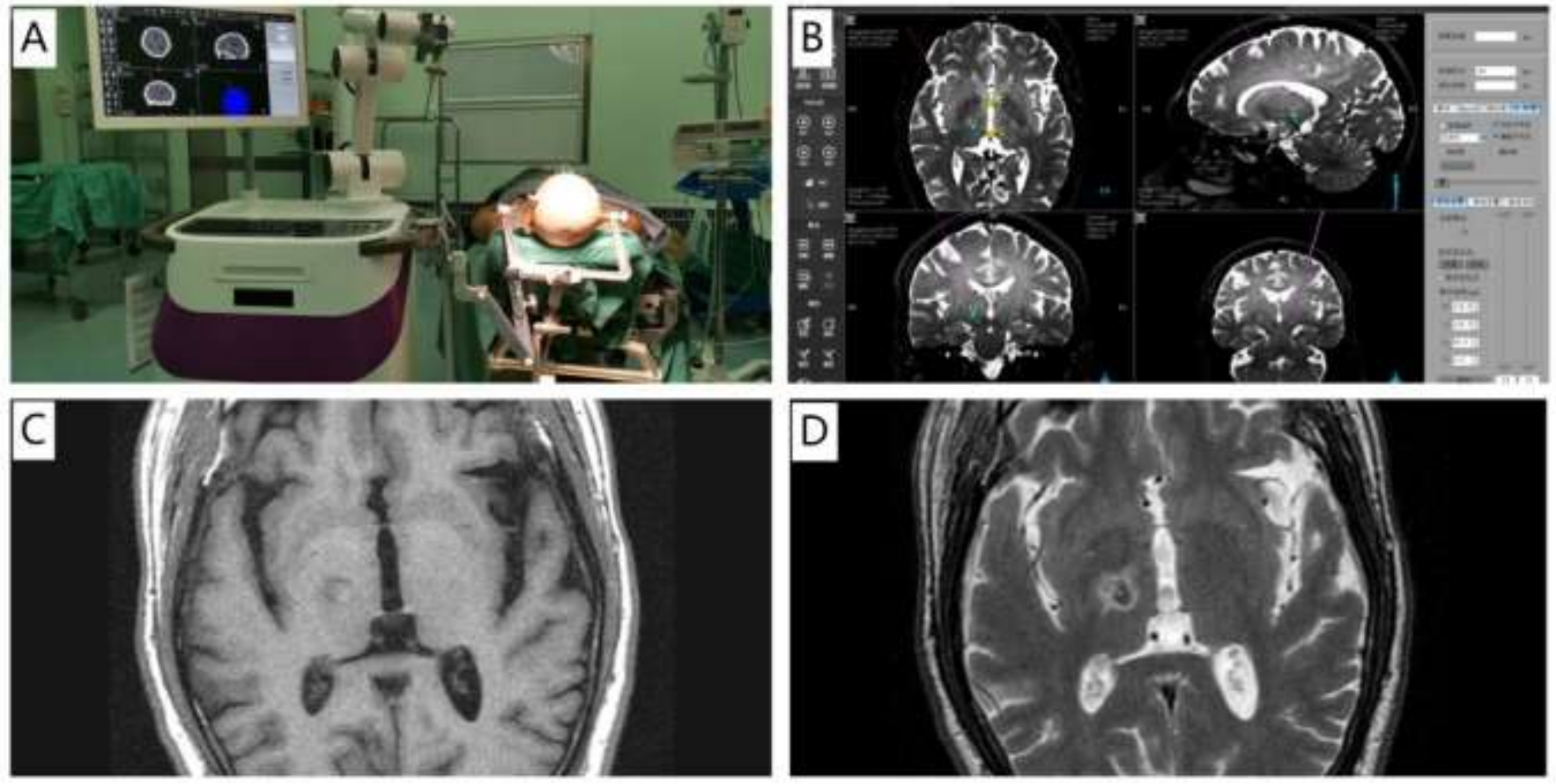

Figure 5. Destructive surgery for Parkinson's disease. A. A fixation device was used to fix the patient, the operating table and the machine; B. Surgical planning; C. Postoperative MRI T1 images of the skull showed the lesion was accurate; D. Postoperative reexamination of brain MRI T1 images showed that the lesion was accurate.

The new CR brain surgery robot system has completed the risk analysis and evaluation of the produ ct according to EN ISO14971. This work includes: (1) The risk management plan has been properly impl emented; (2) The overall residual risk is acceptable; (3) There are appropriate methods to collect pre-IPO and post-IPO risks. All the residual risks of the CR neuro-stereotactic surgery system meet the risk acc 
eptance criteria, the product has obtained the CE registration certificate, and the overall risks of the prod uct are acceptable.

\section{Discussion}

These has always been the goals pursued by the surgeons to reduce the difficulty of operation, increase the accuracy and safety of the operation, reduce the complications of the operation, reduce the trauma, shorten the operation time and improve the comfort of the patients. The emergence of robotic surgery systems is gradually replacing traditional frame surgery and continues to move towards the above goals [18-20]. On the basis of the preliminary research and development and application, we have improved the CAS-R-2 robot in terms of hardware configuration, computer graphics and image processing and human interaction, and developed a new generation of neurosurgical robot system $(\mathrm{CR})$ to make up for the lack of products of the older generation, making it more in line with clinical needs, and 120 operations were successfully performed. Compared with the current domestic active arm robots, it is more flexible and maneuverable. The joint design of the manipulator is closer to human arm joints and the operation of the manipulator is more ergonomic. There are fewer interference problems with other equipment in the operating space, and the work area is wider. The operation of the new generation of neurosurgical CR-2 is simple and easy to learn. So, the learning curve is short. The surgeon can master and use the CR-2 in clinical work after a short-term training. Otherwise, the $\mathrm{CR}$ has a high-cost performance. All parts and components of the project products are $100 \%$ localized, which reduces the overall price of the product, improve the maintainability and the convenience of replacement of parts. All the advantages of the CR lay a good foundation for better popularization of domestic neurosurgery departments.

The new CR brain surgery robot uses MARK points (at least 4) attached to the head to perform CT or MRI scans, and the resulting CT/MRI image data has less than three MARK points on each slice. CT or MRI data were imported into the product system and the space coordinate system was established by the MARK points. The target point of the lesion was marked, and the surgical path was planned in the product system. Then the product system could inversely solve the angle value of each joint by the robotic arm. The end of the robotic arm was moved to the designated position according to the angle value to establish a surgical path, and the needle could be inserted into the patient's lesion target following the path.

The equipment is optimized according to the expected use of the new CR brain surgery robot and the potential safety issues of the CAS-R-2 robot. The new CR brain surgery robot has the following improvements in terms of safety. First, we increase the additional $40 \mathrm{~kg}$ counterweight of the machine itself and the contact area between the counterweight body and the ground. Second, we increase the load of the mechanical arm, which can extend the service life of the $\mathrm{R}$ joint. Third, the external bearing capacity of the mechanical arm is increased by $7 \mathrm{~kg}$ to ensure that the mechanical arm does not deviate when it is drilling. Last, Modular control of the control box is carried out to reduce the impact of the transportation process on the stability of the equipment.

Compared with the CAS-R-2 robot, the new generation robot has higher stability during surgical fixati on due to the increase of its counterweight in terms of hardware. At the same time, the fixing method is improved. One-key lifting is adopted, and the four universal wheels are usually on the ground for easy movement during the free time. During the operation, we can raise the four wheels with one button to $\mathrm{m}$ ake the counterweight flat on the ground, and then the machine is firmly fixed when the machine is in pl ace (Figure 6). The original flexibility of CR-2 robot is maintained while the mechanical stability is improv ed. The new robot system is equipped with two accessories, a shaping pillow and a fixed connection de vice, which are suitable for different types of surgery. Plastic pillow fixation can be used to reduce the $p$ atient's pain, simplify the process and improve the surgical efficiency for biopsy and cerebral hemorrhage drainage performed under general anesthesia. The patient's head is fixed with a stereotactic four-nail hea d frame or Mayfield three-nail head frame, and a robot-specific fixing device is used to fix the head fram e with the operating table and the robot to avoid relative displacement and ensure surgical accuracy whe $\mathrm{n}$ the surgeon is performing operations, such as brain core mass destruction, deep brain electrode stimul ation (DBS) or stereoelectroencephalography (SEEG). 

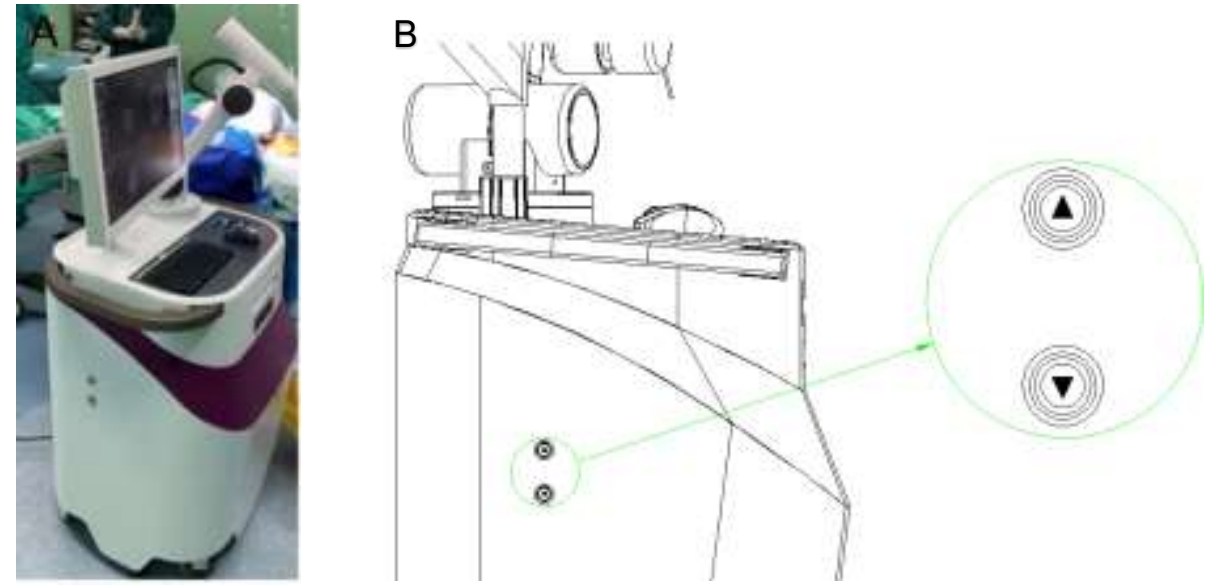

Figure 6. One-key lifting device of the robot.

In terms of software, the human-computer interaction interface has been improved according to the $\mathrm{c}$ linician's suggestions, and the human-computer interaction operation is more reasonable. The display func tion of the slice where the target path is located is increased, so that the surgeon can observe the relev ant structure of the puncture level from multiple angles and measure at this level, which is convenient fo $r$ the surgeon to design the surgical path. We add the functions of three-dimensional section superpositio $\mathrm{n}$, rotation and volume block construction, which can be used to observe the target point and puncture $p$ ath from multiple dimensions, especially when the surgeon is performing surgery in the sellar region and pineal area. These functions can be adopted to assist to observe the relationship between the needle pa th and the peripheral cerebral cisterna and blood vessels, ensuring the safety of the operation. The imag e fusion module is added to realize multi-modal fusion technology (such as: CTA, MRA, MRV and cortic al rendering technology, etc.). It can display details, such as the route of cortical entry into cranial point sulci and adjacent structure of arteries and veins, as well as puncture path, so as to improve surgical sa fety. Functional neurosurgery modules are added to facilitate the design and planning of non-visual target s. Multitarget design modules has been added and be used for operations like SEEG. The design functio $\mathrm{n}$ of the scalp entry point is added, and the surgical path can be designed according to the position of $\mathrm{t}$ he scalp entry point. The above improvements meet the development requirements of precision neurosur gery. The fine-tuning function is also added. Slight errors may occur sometimes during image fusion due to the difference in scanning angle and layer thickness. The fine-tuning function allows the surgeon to $\mathrm{m}$ ake multi-directional fine adjustments on the basis of machine fusion, making the image fusion more acc urate.

The improved CR robot system can be applied to all current types of stereotactic surgery, compared with the previous generation of products, enhancing the practicality of surgery. A total of 120 surgeries were performed in this group, including intracranial lesion biopsy, intracranial hematoma evacuation, OMMAYA capsule implantation, brain abscess aspiration, brain core mass destruction and stereotactic-guided resection of small lesions in the brain. In all cases, the robot could enable us to accurately reach the planned target and provide stable support and guidance for the surgical instruments during the surgical operation stage. We note that frame stereotactic biopsy is still the first choice for the deepest and smallest lesions or lesions near vascular areas, such as the brainstem, sellar area and pineal area in many medical centers. In this group of cases, there were 16 cases of brainstem diseases, 4 cases of sellar region diseases, and 3 cases of pineal region diseases, all of which obtained clear pathological diagnosis without complications, confirming that robotic surgery is also applicable to the surgery of these parts. It is confirmed that robotic surgery is also suitable for surgery in these parts. In this group of cases, 4 cases of brain core mass destruction operations were also completed. Three of them were Parkinson's patients with tremor as the main manifestation. The intraoperative damage needles were in place at one time and the Vim nucleus of the thalamus was damaged. Eventually, the operation effect was good.

Surgical accuracy and complication rate are the primary objectives for evaluating the reliability of stereotactic equipment. The robot surgery still has the possibility of relative displacement compared with the stereotactic 
surgery of the patient's skull fixed frame. Therefore, more attention should be paid to the fixation, registration, and calibration issues in the robot surgery.

We used two fixation methods in the operation, fixation of plastic pillow (Figure 7) and head frame combining the fixed device (Figure 5A). For the operation of visible targets such as biopsy, hematoma, or abscess under general anesthesia, we all adopt the method of plastic pillow fixation. The advantages of this method are easy operating, short time-consuming, less instruments and equipment in the surgical field, less mutual interference, less trauma, and less pain to the patient. The disadvantage is that if you do not pay attention to the fixed details, it is easy to produce relative displacement. We should pay attention to the following points when we are using plastic pillows. First, remove the headrest or sponge cushion and make the plastic pillow directly contact the metal bed. Second, the shaping pillow should fix the head space at least 1/2. Third, the edge of the shaping pillow should wrap the edge of the bed body, so that it is tightly combined with the bed body after suction and shaping to avoid relative displacement. For local anesthesia surgery, fixation of head frame combining the fixed device can be used to fix patients with involuntary movement or invisible or high-precision target surgery such as Vim, Gpi and STN, and multi-target surgery under general anesthesia (such as SEEG surgery). The advantages of this method are that it is firmly fixed, not easy to produce relative displacement and convenient for repeated operation or adjustment. The disadvantages are that the fixation method is complex, the operation time is long, and there are many instruments and devices in the surgical field, which are easy to interfere with each other, and the patient suffers great trauma and pain.

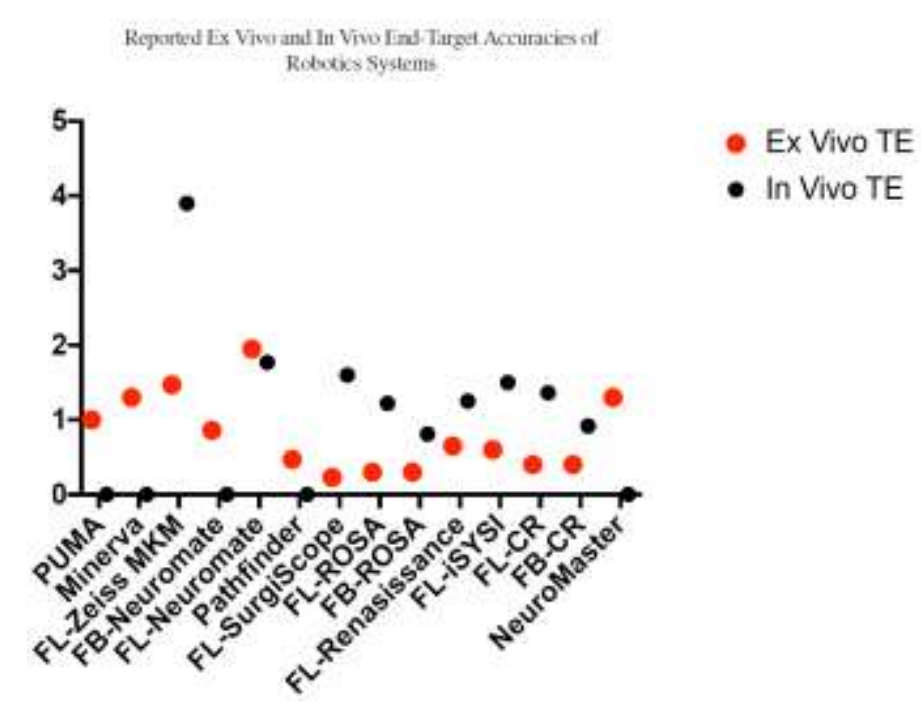

Figure 7. The domestic CR surgical robot is compared with other reported surgical robots in terms of accuracy of the skull entry point and the final target point

Robot registration methods include frame-based registration, frameless base point (skin or bone) re gistration and frameless laser surface registration, which can be selected according to different disease lo cations and types of surgery. In the operation of relatively large visible lesions (such as biopsy, cerebral hemorrhage, and brain abscess), we mainly use frameless scalp fiducial registration. This registration met hod is convenient, fast, and accurate. It only takes about only 2 minutes to register and verify. The follo wing points should be noted when using scalp base points. First, we should use at least 4 registration $p$ oints and the mapping space should include lesions. Second, two additional verification points can be pa sted and verified after registration to prevent errors in scalp displacement during the fixation and registrat ion process. Third, we should try to stick it on the scalp where it is not easy to move when we are stic king the mark. Last, bone benchmark registration is applicable to DBS and SEEG surgeries.

It is reported in the literature that the method of skull self-stop drilling (about $1-1.5 \mathrm{~cm}$ in diameter) is used to drill large bone holes in stereotactic surgery in most of medical centers. This method should have no effect on the accuracy during frame stereotactic surgery. But it is necessary to consider the possibility of relative displacement during drilling in the robot operation. Therefore, we adopt two methods during operation to avoid this situation 
during surgery. First, a dedicated fine drill (about $3-4 \mathrm{~mm}$ in diameter) is used to drill small bone holes in the operation of relatively large visible lesions (such as biopsy, cerebral hemorrhage, and brain abscess). The drill is sharp, so it can reduce the pressure on the skull and the possibility of relative displacement can be reduced. The thickness of the skull can be measured according to the three-dimensional reconstruction image of the workstation and the depth of the skull drill can be controlled by the guide stopper to ensure the safety of the drilling process. Second, the method of secondary registration, the re-registration of fiducial point after drilling the bone hole, is mainly used in the cases of cerebral core mass destruction, DBS or SEEG surgery, which can effectively reduce the possibility of relative displacement.

We measured the accuracy of this group of cases. In 82 cases of biopsy operations, the patients were fixed with a plastic pillow under general anesthesia. Postoperative target accuracy evaluation showed that the target error was $1.36 \pm 0.42 \mathrm{~mm}$. In 4 patients with brain core mass destruction, Mayfield three-nail surgical head frame was used to fix the patient's head during the operation. After the robot was fixed to the surgical head frame with a fixed-link device, the operation was performed under local anesthesia. The postoperative target accuracy evaluation results showed that the target error was $0.92 \pm 0.21 \mathrm{~mm}$. It is reported in the literature that a comparative evaluation of the accuracy of four frame-based in vitro targets shows that the average error is between 1.7 and $1.9 \mathrm{~mm}$ [21] and the average target error is $1.4 \pm 0.9 \mathrm{~mm}$ [33] in a series of frame-based DBS operations. Widmann et al. reported that the average target error of head mold study was between 1.1 and $1.3 \mathrm{~mm}$ and the average target error of real surgery was between 1.99 and $3.2 \mathrm{~mm}$ [22]. Bot et al. compared the accuracy of 194 DBS based on Nexframe and Leksell frame positioning. The results showed that the average target error of Euclidean distance was $2.71 \pm 1.23$ and $2.63+1.07 \mathrm{~mm}$ [23]. Fomenko et al. systematically reviewed the target accuracy comparison of various types of robotic systems in intracranial stereotactic functional surgery under frameless and frame operations. They found that the ex Vivo end-target error ranged from $0.23 \pm 0.13 \mathrm{~mm}$ to $1.95 \pm 0.44 \mathrm{~mm}$ [2432 ] and the in Vivo end-target error ranged from $0.81 \pm 0.39 \mathrm{~mm}$ to $3.3-4.5 \mathrm{~mm}$ [33-47]. We compared the surgical accuracy values of the domestic CR surgical robot system with those in the literature and found that our results are acceptable compared with the traditional frame-based and frameless DBS. (See Figure 5)

The improvement of the system stability and the surgical software ensure the accuracy of the operation while reducing the risks of the operation itself. In this group of 120 patients, the overall surgical success rate was $100 \%$ and the early clinical transient complication rate was $2.5 \%$. One of the biopsy patients had moderate bleeding at the biopsy site, which was improved after treatment. A small amount of bleeding occurred in the operation area in 2 cases with no obvious symptoms. The reasons of bleeding are all related to the vascular-rich characteristics of the tumor itself, not related to the puncture path. The 4 dead patients with brainstem hemorrhage and 2 patients with supratentorial hematoma eventually died due to severe symptoms on admission, but surgical puncture itself did not cause new bleeding.

We also noticed that the operation takes longer when robots were used to complete the above operations in the literature [47-50]. We analyzed the factors that affect the operation time. The first factor is registration method during the operation. Whether the surgical head frame should be installed and connected with the robot system? Whether the space negotiation between the robotic arm and the equipment in the operating area will occur during the movement? Whether the path should be replanned during the operation? The new CR robot is more flexible and maneuverable. Passive arm robots are smaller in size, more flexible in movement, unrestricted in positioning, and can be positioned flexibly according to surgical requirements. The manipulator joint design of the passive arm robot is closer to the human arm joint, and the operation is more ergonomic, with fewer interference problems with other equipment in the operating space and wider working area, especially more convenient in the operation of the posterior cranial fossae. The passive arm robot is easy to operate, easy to register with Mark, fast and efficient, and the operation time is shorter. We usually adopt plastic pillow fixation under general anesthesia in singlechannel surgery, which greatly reduces the fixation time. In conclusion, the operation time is greatly shortened by using the new CR robot (see Table 1).

Of course, we have also found that the operator should pay more attention to more details when completing DBS surgery by using the robot system in clinical applications in terms of design concepts, compared with the framework. And that does not provide more convenience. For patients, the framework cannot be removed, and 
patients also need to accept the pain caused by bone markers, which does not improve the comfort of surgery. There are many areas to be improved. For multi-channel SEEG surgery, the robot is more convenient than the frame system and can greatly shorten the operation time. The new CR robot system can also complete this type of surgery from the design concept compared with the active arm robot. But, compared with active arm robots, there is a gap in the clinical application because the arm joints must be manually adjusted.

\section{CONCLUSION}

In this study, we demonstrate the utility of the improved new CR robot in the treatment of neurosurgery-related diseases. Robots combine human decision-making with the precision of machine technology to improve the safety and feasibility of a variety of minimally invasive surgeries, while minimizing risks and reducing operation time. Compared with the active-arm robots currently used in the market, the CR passive-arm robot has shown its certain advantages, especially suitable for some small-channel operations (such as biopsy, intracerebral hemorrhage, and brain abscess). In addition, it is suitable for promotion in hospitals at all levels because of its high-cost performance. At the same time, we need to carry out more case studies to validate the previous results, improve the current technology and optimize the impact of the robotic stereotactic system on the quality of neurosurgery.

\section{REFERENCES}

1. Barbash, Gabriel, I., Glied, Sherry A. New technology and health care costs - the case of robot-assisted surge ry. N. Engl. J. Med. 363:701-704, (2010).

2. Bergeles, C., and G. Yang. From passive tool holders to microsurgeons: safer, smaller, smarter surgical robot s. IEEE Trans. Biomed. Eng. 61:1565-1576, (2014).

3. Gerber N, K. A. Gavaghan, B. J. Bell, T. M. Williamson, C. Weisstanner, M.-D. Caversaccio, et al. High-ac curacy patient-to-image registration for the facilitation of image-guided robotic microsurgery on the head. IEE E Trans. Biomed. Eng. 60:960-968, (2013).

4. Kwoh, Y. S., L. S. Reed, J. Y. Chen, H. Shao, T. K. Truong, and E. A. Jonckheere. A new computerized t omographic aided robotic stereotactic system. Robot. Age 7:17-21, (1985).

5. Adler JR Jr, Murphy MJ, Chang SD, Hancock SL: Image- guided robotic radiosurgery. Neurosurgery 44:1299 -1307, (1999).

6. BALLANTYNE GH. Robotic surgery, telerobotic surgery, telepresence, and telementoring: Review of early cli nical results[J]. Surg Endosc,16:1389-1042. (2002)

7. Woodworth GF, McGirt MJ, Samdani A, Garonzik I, Olivi A, Weingart JD: Frameless image-guided stereotac tic brain biopsy procedure: diagnostic yield, surgical morbidity, and comparison with the frame-based techniqu e. J Neurosurg 104:233-237, (2006).

8. Sutherland, G. R., S. Wolfsberger, S. Lama, and K. Zareinia. The evolution of NeuroArm. Neurosurgery72:A 27- A32, (2013).

9. Narendra Nathoo, M Cenk Cavuşoğlu, Michael A Vogelbaum, Gene H Barnett. In Touch With Robotics: Neu rosurgery for the Future. Neurosurgery Mar;56(3):421-33. (2005)

10. Shenai MB, Tubbs RS, Guthrie BL, Cohen-Gadol AA: Virtual interactive presence for realtime, long-distance surgical collaboration during complex microsurgical procedures. J Neurosurg. 121:277-284. (2014)

11. Korte C, Nair SS, Nistor V, Low TP, Doarn cr, Schaffner G: Determining the threshold of time-delay for tel eoperation accuracy and efficiency in relation to telesurgery. Telemed J E Health. 20:1078-108. (2014)

12. TIAN Zengmin, ZHAO Quanjun, Du Jixiang, et al. Use of robot in frameless stereotactic neurosurgery. Chin J Minim Invasive Neurosurg, 5(3): 129-130. (2000)

13. TIAN Zeng-min, ZHAO Quan-jun, YU Xin. Clinical Report of 5100 Cases with Stereotactic Operation. Jour nal of Naval General Hospital. 20 (2): 65-67.(2007)

14. YIN Feng, TIAN Zengmin, WANG Tianmiao, et al. Clinical application of the fifth robotic system in neuros urgery. Chin J Minim Invasive Neurosurg, 13(8): 355-357. (2008) ) 
15. Zeng-min Tian, Wangsheng Lu, Tianmiao Wang, Belin Ma, Quanjun Zhao, Guolai Zhang. Application of a $r$ obotic telemanipulation system in stereotactic surgery. Stereotact Funct Neurosurg, 86(1):54-61. (2008) )

16. Zeng-Min Tian, Wang-Sheng Lu, Tian-Miao Wang, Du Liu, Yan Chen, Guo-Lai Zhang, et al. Clinical applic ation of robotic tele-manipulation system in stereotactic surgery. Zhonghua Wai Ke Za Zhi. Dec 15;45(24):167 9-81. (2007)

17. Zeng-min Tian, Wang-sheng Lu, Quan-jun Zhao, Xin Yu, Shu-bin Qi, Rui Wang. From Frame to Framless $\mathrm{S}$ tereotactic Operation - Clinical Application of 2011 Cases. International Conference on Medical Imaging and I nformatics MIMI: Medical Imaging and Informatics pp. 18-24. (2007)

18. Dammers R, Haitsma IK, Schouten JW, Kros JM, Avezaat CJJ, Vincent AJPE: Safety and effic acy of frameless and frame-based intracranial biopsy techniques. Acta Neurochir (Wien) 150:2329, (2008)

19. Dorward NL, Alberti O, Palmer JD, Kitchen ND, Thomas DG: Accuracy of true frameless stereotaxy: in viv o measurement and laboratory phantom studies. Technical note. J Neu- rosurg 90:160-168, (1999).

20. Woodworth GF, McGirt MJ, Samdani A, Garonzik I, Olivi A, Weingart JD: Frameless image-g uided stereotactic brain biopsy procedure: diagnostic yield, surgical morbidity, and comparison $\mathrm{w}$ ith the frame-based technique. J Neurosurg 104:233-237, (2006).

21. Maciunas RJ, Galloway RL Jr, Latimer JW: The application accuracy of stereotactic frames. Ne urosurgery 35:682-695, (1994).

22. Widmann G, Schullian R Ortler M, Bale R: Frameless stereotactic targeting devices: technical features, targeti ng errors and clinical results. Int J Med Robot 8:1-16, (2012).

23. Bot M, van den Munckhof P, Bakay R, Sierens D, Stebbins G, Verhagen Metman L: Analysis of stereotactic accuracy in patients undergoing deep brain stimulation using Nexframe and the Leksell frame. Stereotact Fu nct Neurosurg 93:316-325, (2015).

24. A novel miniature robotic device for frameless implantation of depth electrodes in refractory epilepsy [J]. Chi $n$ J Neurosurg Dis Res, 126(4): 308. (2016)

25. AMIN D V, LUNSFORD L D. Volumetric resection using the SurgiScope: a quantitative accuracy analysis o f robot-assisted resection [J]. Stereotactic \& Functional Neurosurgery, 82(5-6): 250-3. (2004)

26. Barati M, KHOOGAR A R, NASIRIYAN M. Reduce Positioning Error for Robot Manipulator with Intelligen t Technique [J]. Advanced Materials Research,403-408(697-706). (2011)

27. Bekelis K, Radwan T A, Desai A, et al. Frameless robotically targeted stereotactic brain biopsy: feasibility, d iagnostic yield, and safety [J]. Journal of Neurosurgery, 116(5): 1002. (2012)

28. DE Benedictis A, Trezza A, CARAI A, et al. Robot-assisted procedures in pediatric neurosurgery [J]. Neuros urg Focus, 42(5): E7. (2017)

29. Deblaise D, Maurine P. Effective geometrical calibration of a delta parallel robot used in neurosurgery; proce edings of the IEEE/RSJ International Conference on Intelligent Robots \& Systems, F, 2005 [C].

30. Eljamel, M. S. Robotic neurological surgery applications: accuracy and consistency or pure fantasy? [J]. Stere otactic \& Functional Neurosurgery, 87(2): 88-93. (2009)

31. Eljamel M S. Validation of the PathFinder neurosurgical robot using a phantom [J]. International Journal of Medical Robotics \& Computer Assisted Surgery, 3(4): 372-7. (2007)

32. Fomenko A, Serletis D. Robotic Stereotaxy in cranial Neurosurgery: A Qualitative Systematic Review [J]. Ne urosurgery, 83(4): 642-50. (2018)

33. Glauser D, Fankhauser H, Epitaux M, et al. Neurosurgical robot Minerva: first results and current developme nts [J]. J Image Guid Surg,1(5): 266-72. (1995)

34. Grimm F, Naros G, Gutenberg A, et al. Blurring the boundaries between frame-based and frameless stereotax y: feasibility study for brain biopsies performed with the use of a head-mounted robot [J]. Journal of Neuros urgery, (2015).

35. Guenot, MARC. Stereoelectroencephalography: Surgical Methodology, Safety, and Stereotactic Application Acc uracy in 500 Procedures COMMENT [J]. Neurosurgery, (2013). 
36. Hefti J L, Epitaux M, Glauser D, et al. Robotic three-dimensional positioning of a stimulation electrode in th e brain [J]. Comput Aided Surg, 3(1): 1-10. (1998)

37. Joskowicz L, Shamir R R, Israel Z, et al. Renaissance Robotic System for Keyhole cranial Neurosurgery: Invitro Accuracy Study [J].

38. Kwoh Y S, Hou J, Jonckheere E A, et al. A robot with improved absolute positioning accuracy for CT guid ed stereotactic brain surgery [J]. IEEE Trans Biomed Eng, 35(2): 153-60. (1988)

39. Lefranc M, Capel C, A.S. P, et al. The Impact of the Reference Imaging Modality, Registration Method and Intraoperative Flat-Panel Computed Tomography on the Accuracy of the ROSA® Stereotactic Robot [J]. Stere otactic \& Functional Neurosurgery, 92(4): 242-50. (2014)

40. Lefranc M, Gars D L. Robotic implantation of deep brain stimulation leads, assisted by intra-operative, flat-p anel CT [J]. Acta Neurochirurgica, 154(11): 2069-74. (2014)

41. Li Q H, Zamorano L, Pandya A, et al. The application accuracy of the NeuroMate robot-A quantitative co mparison with frameless and frame-based surgical localization systems [J]. Computer Aided Surgery, 7(2): 908. (2002)

42. Liu J, Zhang Y, LI Z. The Application Accuracy of NeuroMaster: a Robot System for Stereotactic Neurosurg ery; proceedings of the IEEE/ASME International Conference on Mechatronic \& Embedded Systems \& Applic ations, F, 2007 [C].

43. Minchev G, Kronreif G, MARTÍNEZ-MORENO M, et al. A novel miniature robotic guidance device for ster eotactic neurosurgical interventions: preliminary experience with the iSYS1 robot [J]. Journal of Neurosurgery, 1-12. (2016)

44. SPINEASSIST. Mazor Robotics Launches Renaissance(tm), the Next Generation of Its Highly Accurate Roboti c Surgical Guidance Systems [J]. Biomedical Market Newsletter, (2011),

45. Von Langsdorff D, Paquis P, Fontaine D. In vivo measurement of the frame-based application accuracy of th e Neuromate neurosurgical robot [J]. Journal of Neurosurgery, 122(1): 191. (2015)

46. Willems P W, Noordmans H J, Ramos L M, et al. Clinical evaluation of stereotactic brain biopsies with an MKM-mounted instrument holder [J]. Acta Neurochir (Wien), 145(10): 889-97; discussion 97. (2003)

47. Willems P W A, Noordmans H J, Van der sprenkel J W B, et al. An MKM-mounted instrument holder for $\mathrm{f}$ rameless point-stereotactic procedures: a phantom-based accuracy evaluation [J]. Journal of Neurosurgery, 95 (6): 1067. (2001)

48. Candela S, Vanegas M I, Darling A, et al. Frameless robot-assisted pallidal deep brain stimulation surgery in pediatric patients with movement disorders: precision and short-term clinical results [J]. J Neurosurg Pediatr, 22(4): 416-25. (2018)

49. De benedictis A, Trezza A, Carai A, et al. Robot-assisted procedures in pediatric neurosurgery [J]. Neurosurg ical Focus, 42(5): E7. (2017)

50. Vansickle D, Volk V, Freeman P, et al. Electrode Placement Accuracy in Robot-Assisted Asleep Deep Brain Stimulation [J]. Annals of Biomedical Engineering, 2019. 


\section{Declaration Of Patient Consent}

The authors certify that they have obtained all appropriate patient consent forms. In the form, the patient has given his consent for his images and other clinical information to be reported in the journal. The patient understands that his name and initial will not be publish ed and due efforts will be made to conceal his identity, but anonymity cannot be guaranteed.

\section{Author Contributions}

FY: concept and protocol development, writing and review of the manuscript. HC, XY, S-CD, M-M Z, S-MC, TL, C-JY, YL: data collection, sample collection, research plan, performing research. YH, FZ: statistical analysis. Y-MW: supervision and critical review of the manuscript.

\section{Funding}

\section{National Natural Science Foundation of China (NO:62171003)}

\section{Competing interests}

The authors declare no competing interests.

Data availability

The datasets generated during and/or analyzed during the current study are available from the corresponding author on reasonable request.

\section{Additional information}

Correspondence and requests for materials should be addressed to F.Y. 

